\title{
The influence of RhD phenotype on toxoplasmosis- and age-associated changes in personality profile of blood donors
}

\author{
Jaroslav Flegr ${ }^{1}$, Martina Novotná ${ }^{1}$, Anna Fialová ${ }^{1}$, Petra Kolbeková $^{1}$ and Zdeňka Gašová $^{2}$ \\ ${ }^{1}$ Biology Section, Faculty of Science, Charles University, Viničná 7, 12844 Prague, Czech Republic; \\ ${ }^{2}$ Institute of Haematology and Blood Transfusion, U nemocnice 1, 12000 Prague, Czech Republic
}

\begin{abstract}
Toxoplasma gondii, a parasite infecting 20-60\% of humans in various countries, influences the behaviour of infected animal and human hosts. Infected human subjects have changes in several of Cattell's and Cloninger's personality factors. Recently, three independent studies have shown that Rh-positive subjects are protected against the $T$. gondii-induced changes of reaction times and increased risk of traffic accidents. Here we searched for evidence of similar effects of RhD phenotype on toxoplasmosis- or aging-associated changes in the personality profile of about 302 blood donors. We found that Rh-positive and Rh-negative subjects responded differently to toxoplasmosis. In addition to the already known effects of toxoplasmosis on novelty seeking, self transcendence, superego strength and protension, we also found effects of $\mathrm{RhD}$ phenotype on ego strength, protension, and praxernia, as well as opposite effects of toxoplasmosis on ego strength, praxernia, ergic tension and cooperativeness in Rh-positive and Rh-negative subjects. Moreover, our results indicate that $\mathrm{RhD}$ phenotype might influence not only the effect of toxoplasmosis but also the effect of aging on specific personality traits.
\end{abstract}

Keywords: parasite, manipulation, personality, blood group, Rh factor, Cattell 16PF, Cloninger TCI, Rhesus factor, aging, senescence

The RhD protein which is the RHD gene product and a major component in the $\mathrm{Rh}$ blood group system carries the strongest blood group immunogen, the $\mathrm{D}$ antigen (Carritt et al. 1997, Flegel 2006). This antigen is absent in a significant minority (about 16\%) of the Caucasian population (Rh-negatives) due to $R H D$ deletion or alternation. The structure homology data suggest that the $\mathrm{RhD}$ protein acts as an ion pump of uncertain specificity and unknown physiological role (Biver et al. 2006, Kustu and Inwood 2006). Except for the well known but artificial role of the $\mathrm{RhD}$ protein in haemolytic disease of newborns, no phenotypic expression of the absence or presence of $\mathrm{RhD}$ on the surface of erythrocytes of a subject was known for more than sixty years. Recently, two studies on two populations of blood donors, one of conscripts (Novotná et al. 2008) and the other of university students (Flegr et al. 2008), have shown that Rh-positive subjects, and RhDpositive heterozygotes in particular, are protected against latent toxoplasmosis-induced impairment of reaction times. A prospective cohort study performed on nearly four thousand military drivers has found that Rh-negative Toxoplasma-infected subjects have about three times higher probability of a traffic accident than $\mathrm{Rh}$-negative Toxoplasma-free subjects or than Rh-positive (Toxoplasma-free or Toxoplasma-infected) subjects (Flegr et al.
2009). Another study performed on 980 pregnant women has found the RhD-positivity protects Toxoplasma-infected women against excessive weight gain in the first trimester of pregnancy (Kaňková et al. 2010).

The protozoan parasite Toxoplasma gondii infects $20-60 \%$ of the population in most countries, depending on climate, hygienic standards and cooking habits (Tenter et al. 2000). Postnatally acquired toxoplasmosis in immunocompetent subjects causes usually only mild disease, acute toxoplasmosis, which turns spontaneously into lifelong latent toxoplasmosis. Latent toxoplasmosis is characterized by the presence of the dormant cyst stage of the parasite mainly in the neural and muscular tissues and immunity against new Toxoplasma infections (Remington and Krahenbuhl 1982, Jones et al. 2001). Latent toxoplasmosis in humans is considered as clinically asymptomatic (Markell et al. 1999, Roberts and Janovy 2000). However, infected people seem to have impaired reaction times (Havlíček et al. 2001) and a higher risk of traffic accidents (Flegr et al. 2002, Yereli et al. 2006, Kocazeybek et al. 2009), possibly as a result of manipulation activity of $T$. gondii "aimed" to increase the chance of transmission from the intermediate to the definitive host, i.e. from any bird or mammal species to any feline species, by predation. Between 1994 and 2007, about twelve 
studies have reported that Toxoplasma-infected men and women differed in several personality factors measured with Cattell's 16PF questionnaire or Cloninger's TCI questionnaire from Toxoplasma-free subjects (see Flegr 2007 for review). The difference in personality traits increases with the duration of the infection, which suggests that the changes are induced by toxoplasmosis rather than that the risk of Toxoplasma infection is influenced by the personality profile of subjects (Flegr et al. 2000). Moreover, the results of about forty years of research performed on laboratory animals have shown that $T$. gondii is able to specifically influence the behaviour and temper of its intermediate hosts (Webster 2001), probably by regulating the concentration of neurotransmitters, dopamine and possibly also serotonin, in particular regions of the brain (Skallová et al. 2006, Hodková et al. 2007). It has even been recently reported that the genome of $T$. gondii contains two genes for aromatic amino acid hydroxylases, the enzymes not previously described in the protozoa, and these enzymes probably catalyze the rate-limiting step of the biosynthesis of dopamine in the brain of infected hosts (Gaskell et al. 2009).

The aims of the present study were 1) to find out whether Rh-positive subjects are protected not only against the impairment of reaction times and excessive weight gain in pregnancy but also against the toxoplasmosis-associated specific changes in personality traits and 2) to find whether RhD phenotype modifies not only the host response to Toxoplasma infection but also to other biological factors, namely the age of the host.

\section{MATERIALS AND METHODS}

Subjects. During the thrombocyte separation sessions, about eight hundred donors of thrombocytes at the blood transfusion centres of the Institute of Haematology and Blood Transfusion, Prague, and of the Zbraslav hospital, Prague, were asked to voluntarily participate in the research project and to sign the informed consent form. Five hundred and thirty-one (about 70\%) of them consented to provide their psychological data and $5 \mathrm{ml}$ of blood for serological examination. They were given the Cloninger's TCI and Cattell's 16PF questionnaire forms and a stamped envelope with the address of the Department of Parasitology, Faculty of Science, Charles University on it. Three hundred and two TCI questionnaire forms (57.2\%) and $26216 \mathrm{PF}$ questionnaire forms $(49.4 \%)$ were filled in and returned. The recruitment of subjects and the data handling complied with the applicable regulations.

Personality tests. TCI (Temperament and Character Inventory) (Cloninger et al. 1994): The Czech translation of the TCI questionnaire (Kožený and Tisanská 1998) was used, with the translation of two questions being corrected and Cloninger's validation scale being substituted with Eysenck's Lie scale EP/R. The final questionnaire (Preiss and Klose 2003) contained all 238 TCI items and 12 Lie scale items. The subjects received written instructions to answer YES or NO for each item depending on whether or not the sentence in the questionnaire characterized their usual and most probable behaviour or feelings in the given situations.
Serological tests. Serological tests for toxoplasmosis were carried out in the National Reference Laboratory for Toxoplasmosis of the National Institute of Public Health, Prague, and serological tests for $\mathrm{RhD}$ and $\mathrm{ABO}$ blood group type were performed at the Institute of Haematology and Blood Transfusion, Prague. Specific anti-Toxoplasma IgG and IgM antibody concentrations were determined by ELISA (IgG: SEVAC, Prague; IgM: TestLine, Brno) optimized for early detection of acute toxoplasmosis (Pokorný et al. 1989) and by the complement fixation test (CFT) (SEVAC, Prague) which is more sensitive and therefore more suitable for the detection of old $T$. gondii infection (Warren and Sabin 1942). Titres of antibodies to Toxoplasma in sera were measured at dilutions between 1:8 and 1:1024. The subjects with negative results of IgM ELISA (positivity index $<0.9$ ) and CFT titres higher than 1:8 were considered latent-toxoplasmosis positive.

Statistical analysis. The software Statistica ${ }^{\circledR}$ version 9.0 was used for General Linear Models (GLM) tests, log-linear analysis and for testing the assumptions, namely those of the normality of data distribution, normality of residuals and equality of error variances.

\section{RESULTS}

Three hundred and two blood donors (213 men and 89 women) were tested for specific immunity against Toxoplasma. The prevalence rates of Toxoplasma infection in men and women were $32.9 \%$ and $27.0 \%$, respectively, and the rates of Rh-negative phenotype were $26.9 \%$ and $22.4 \%$, respectively. The very high proportion of Rh-negative subjects reflected the higher demand for Rh-negative blood and therefore preferential selection of Rh-negative donors rather than the frequency of the $R H D$ deletion in the general Czech population. No association between sex, RhD phenotype, ABO phenotype and Toxoplasma infection was found by the log-linear analysis.

\section{Cloninger's TCI}

The multivariate GLM with toxoplasmosis, RhD phenotype and age as three independent factors and with seven Cloninger's factors as dependent variables showed significant effects of age $(p<0.0001), R h D$ phenotype $(\mathrm{p}=0.011)$, toxoplasmosis $(\mathrm{p}=0.011)$ and toxoplasmosis$\mathrm{RhD}$ interaction $(\mathrm{p}=0.001)$. The univariate GLM analyses showed the effect of toxoplasmosis on novelty seeking (NS) $(p=0.048)$ and self transcendence (ST) $(p=0.024)$, the effect of RhD phenotype on harm avoidance (HA) $(p=0.036)$ and cooperativeness $(\mathrm{CO})(\mathrm{p}=0.009)$ and the effect of toxoplasmosis-RhD interaction on reward dependence $(\mathrm{RD})(\mathrm{p}=0.046)$ and cooperativeness $(\mathrm{CO})$ $(p=0.007)$. For the strength and direction of the effects, see Table 1. The multivariate GLM analysis performed on a subset of men showed the effect of age $(p<0.0001)$, a non-significant effect of toxoplasmosis $(p=0.061)$ and the effect of RhD phenotype $(\mathrm{p}=0.013)$ and of toxoplasmosis-RhD interaction $(\mathrm{p}<0.0001)$. The multivariate GLM analysis performed on a subset of women found the effect of age $(p=0.007)$ and no significant effect of 
Flegr et al.: RhD phenotype and toxoplasmosis-associated changes in personality

Table 1. Effects of latent toxoplasmosis and RhD phenotype on Cloninger's personality factors. The table shows the significance (two-sided $\mathrm{p}$ ), effect size $\left(\eta^{2}\right)$ estimated by the univariate GLM and arithmetic means in particular subsets. The results significant in two-tailed tests are printed in bold. NS - novelty seeking, HA - harm avoidance, RD - reward dependence, SD - self-directedness, $\mathrm{CO}$ - cooperativeness, $\mathrm{ST}$ - self-transcendence, $\mathrm{PE}$ - persistence.

\begin{tabular}{|c|c|c|c|c|c|c|c|c|c|c|c|c|c|c|c|c|c|c|c|}
\hline & \multicolumn{6}{|c|}{ All } & \multicolumn{6}{|c|}{ Men } & \multicolumn{6}{|c|}{ Women } \\
\hline & & $\mathrm{p}$ & $\eta^{2}$ & $\begin{array}{l}\text { toxo- } \\
\mathrm{RhD}^{-}\end{array}$ & $\begin{array}{l}\text { toxo- } \\
\mathrm{RhD}^{+}\end{array}$ & $\begin{array}{l}\text { toxo }^{+} \\
\text {RhD }^{-}\end{array}$ & $\begin{array}{l}\text { toxo }^{+} \\
\mathrm{RhD}^{+}\end{array}$ & $\mathrm{p}$ & $\eta^{2}$ & $\begin{array}{l}\text { toxo- } \\
\mathrm{RhD}^{-}\end{array}$ & $\begin{array}{l}\text { toxo }^{-} \\
\mathrm{RhD}^{+}\end{array}$ & $\begin{array}{l}\text { toxo }^{+} \\
\mathrm{RhD}^{-}\end{array}$ & $\begin{array}{l}\text { toxo }^{+} \\
\mathrm{RhD}^{+}\end{array}$ & $\mathrm{p}$ & $\eta^{2}$ & $\begin{array}{l}\text { toxo- } \\
\mathrm{RhD}^{-}\end{array}$ & $\begin{array}{l}\text { toxo }^{-} \\
\mathrm{RhD}^{+}\end{array}$ & $\begin{array}{l}\text { toxo }^{+} \\
\mathrm{RhD}^{-}\end{array}$ & $\begin{array}{l}\text { toxo }^{+} \\
\mathrm{RhD}^{+}\end{array}$ \\
\hline \multirow[t]{3}{*}{ NS } & toxo & 0.048 & 0.01 & 20.240 & 19.772 & 20.273 & 16.479 & 0.505 & 0.00 & 18.706 & 19.087 & 20.316 & 16.451 & 0.050 & 0.047 & 23.50 & 21.30 & 20.00 & 16.55 \\
\hline & $\mathrm{RhD}$ & 0.053 & 0.01 & & & & & 0.256 & 0.01 & & & & & 0.153 & 0.025 & & & & \\
\hline & toxo $\times \mathrm{RhD}$ & 0.195 & 0.01 & & & & & 0.109 & 0.01 & & & & & 0.875 & 0.00 & & & & \\
\hline \multirow[t]{3}{*}{ HA } & toxo & 0.136 & 0.01 & 14.860 & 14.430 & 17.545 & 14.521 & 0.098 & 0.01 & 14.059 & 14.427 & 18.211 & 13.706 & 0.729 & 0.00 & 16.563 & 14.435 & 13.333 & 16.600 \\
\hline & $\mathrm{RhD}$ & 0.036 & 0.02 & & & & & 0.030 & 0.02 & & & & & 0.802 & 0.00 & & & & \\
\hline & toxo $\times \mathrm{RhD}$ & 0.094 & 0.01 & & & & & 0.013 & 0.03 & & & & & 0.272 & 0.02 & & & & \\
\hline \multirow[t]{3}{*}{$\mathrm{RD}$} & toxo & 0.250 & 0.00 & 14.320 & 15.168 & 15.864 & 14.817 & 0.020 & 0.03 & 13.324 & 14.602 & 16.053 & 14.510 & 0.255 & 0.02 & 16.438 & 16.435 & 14.667 & 15.600 \\
\hline & $\mathrm{RhD}$ & 0.715 & 0.00 & & & & & 0.636 & 0.00 & & & & & 0.701 & 0.00 & & & & \\
\hline & toxo $\times \mathrm{RhD}$ & 0.046 & 0.01 & & & & & 0.008 & 0.03 & & & & & 0.748 & 0.00 & & & & \\
\hline \multirow[t]{3}{*}{ SD } & toxo & 0.502 & 0.00 & 27.420 & 26.510 & 25.955 & 29.338 & 0.828 & 0.00 & 28.471 & 26.155 & 25.789 & 29.216 & 0.418 & 0.01 & 25.188 & 27.304 & 27.000 & 29.650 \\
\hline & $\mathrm{RhD}$ & 0.330 & 0.00 & & & & & 0.882 & 0.00 & & & & & 0.338 & 0.01 & & & & \\
\hline & toxo $\times \mathrm{RhD}$ & 0.069 & 0.01 & & & & & 0.032 & 0.02 & & & & & 0.943 & 0.00 & & & & \\
\hline \multirow[t]{3}{*}{$\mathrm{CO}$} & toxo & 0.147 & 0.01 & 29.260 & 29.181 & 25.727 & 30.254 & 0.771 & 0.00 & 28.412 & 28.262 & 25.474 & 30.588 & 0.154 & 0.03 & 31.063 & 31.239 & 27.333 & 29.400 \\
\hline & $\mathrm{RhD}$ & 0.010 & 0.02 & & & & & 0.018 & 0.03 & & & & & 0.545 & 0.00 & & & & \\
\hline & toxo $\times \mathrm{RhD}$ & 0.007 & 0.02 & & & & & 0.011 & 0.03 & & & & & 0.565 & 0.00 & & & & \\
\hline \multirow[t]{3}{*}{ ST } & toxo & 0.024 & 0.02 & 15.740 & 14.329 & 13.318 & 12.746 & 0.115 & 0.01 & 14.765 & 14.282 & 12.526 & 13.333 & 0.530 & 0.00 & 17.813 & 14.435 & 18.333 & 11.250 \\
\hline & $\mathrm{RhD}$ & 0.335 & 0.00 & & & & & 0.766 & 0.00 & & & & & 0.008 & 0.08 & & & & \\
\hline & toxo $\times \mathrm{RhD}$ & 0.520 & 0.00 & & & & & 0.451 & 0.00 & & & & & 0.379 & 0.01 & & & & \\
\hline \multirow[t]{3}{*}{ PE } & toxo & 0.929 & 0.00 & 4.400 & 4.638 & 4.727 & 4.366 & 0.535 & 0.00 & 4.618 & 4.553 & 4.579 & 4.196 & 0.225 & 0.02 & 3.938 & 4.826 & 5.667 & 4.800 \\
\hline & RhD & 0.936 & 0.00 & & & & & 0.596 & 0.00 & & & & & 0.985 & 0.00 & & & & \\
\hline & toxo $\times \mathrm{RhD}$ & 0.389 & 0.00 & & & & & 0.726 & 0.00 & & & & & 0.237 & 0.02 & & & & \\
\hline $\mathrm{n}$ & & & & 50 & 149 & 22 & 71 & & & 34 & 103 & 19 & 51 & & & 16 & 46 & 3 & 20 \\
\hline
\end{tabular}

Table 2. Effects of latent toxoplasmosis on Cloninger's personality factors. The table shows the significance (two-sided p), effect size $\left(\eta^{2}\right)$ estimated by the univariate GLM and arithmetic means in particular subsets. The results significant in two-tailed tests are printed in bold. For abbreviations see Table 1.

\begin{tabular}{|c|c|c|c|c|c|c|c|c|c|c|c|c|}
\hline & \multicolumn{4}{|c|}{ All } & \multicolumn{4}{|c|}{ Men } & \multicolumn{4}{|c|}{ Women } \\
\hline & $\mathrm{p}$ & $\eta^{2}$ & toxo- & toxo $^{+}$ & $p$ & $\eta^{2}$ & toxo- & toxo ${ }^{+}$ & $\mathrm{p}$ & $\eta^{2}$ & toxo- & toxo $^{+}$ \\
\hline NS & 0.002 & 0.033 & 19.889 & 17.372 & 0.112 & 0.012 & 18.937 & 17.500 & 0.001 & 0.115 & 21.985 & 17.000 \\
\hline HA & 0.411 & 0.002 & 14.548 & 15.319 & 0.557 & 0.002 & 14.336 & 14.929 & 0.510 & 0.005 & 15.015 & 16.458 \\
\hline RD & 0.910 & 0.000 & 14.942 & 15.043 & 0.247 & 0.006 & 14.301 & 14.929 & 0.195 & 0.019 & 16.354 & 15.375 \\
\hline SD & 0.084 & 0.010 & 26.793 & 28.457 & 0.167 & 0.009 & 26.769 & 28.286 & 0.251 & 0.015 & 26.846 & 28.958 \\
\hline $\mathrm{CO}$ & 0.955 & 0.000 & 29.202 & 29.191 & 0.327 & 0.005 & 28.301 & 29.200 & 0.157 & 0.023 & 31.185 & 29.167 \\
\hline ST & 0.016 & 0.019 & 14.750 & 12.872 & 0.134 & 0.011 & 14.483 & 13.114 & 0.034 & 0.051 & 15.338 & 12.167 \\
\hline PE & 0.665 & 0.001 & 4.572 & 4.447 & 0.386 & 0.004 & 4.559 & 4.300 & 0.533 & 0.005 & 4.600 & 4.875 \\
\hline $\mathrm{n}$ & & & 208 & 94 & & & 143 & 70 & & & 65 & 24 \\
\hline
\end{tabular}

Table 3. Effects of RhD phenotype on Cloninger's personality factors. The table shows the significance (two-sided p), effect size ( $\eta^{2}$ ) estimated by the univariate GLM and arithmetic means in particular subsets. The results significant in two-tailed tests are printed in bold. For abbreviations see Table 1.

\begin{tabular}{|c|c|c|c|c|c|c|c|c|c|c|c|c|}
\hline & \multicolumn{4}{|c|}{ All } & \multicolumn{4}{|c|}{ Men } & \multicolumn{4}{|c|}{ Women } \\
\hline & $\mathrm{p}$ & $\eta^{2}$ & $\mathrm{RhD}^{-}$ & $\mathrm{RhD}^{+}$ & $\mathrm{p}$ & $\eta^{2}$ & $\mathrm{RhD}^{-}$ & $\mathrm{RhD}^{+}$ & $\mathrm{p}$ & $\eta^{2}$ & $\mathrm{RhD}^{-}$ & $\mathrm{RhD}^{+}$ \\
\hline NS & 0.113 & 0.009 & 20.289 & 18.733 & 0.491 & 0.002 & 19.404 & 18.252 & 0.040 & 0.051 & 22.947 & 19.864 \\
\hline HA & 0.099 & 0.009 & 15.711 & 14.443 & 113 & 0.012 & 15.596 & 14.1 & 0.606 & 0.003 & 16.053 & 15.091 \\
\hline RD & 0.794 & 0.000 & 14.895 & 15.068 & 0.956 & 0.000 & 14.474 & 14.594 & 0.957 & 0.000 & 16.158 & 16.182 \\
\hline SD & 0.707 & 0.000 & 26.868 & 27.389 & 0.616 & 0.001 & 27.333 & 27.123 & 0.172 & 0.023 & 25.474 & 28.015 \\
\hline $\mathrm{CO}$ & 0.092 & 0.010 & 28.184 & 29.507 & 0.117 & 0.012 & 27.421 & 29.006 & 0.912 & 0.000 & 30.474 & 30.682 \\
\hline ST & 0.219 & 0.005 & 14.868 & 13.787 & 0.853 & 0.000 & 13.860 & 13.923 & 0.003 & 0.101 & 17.895 & 13.470 \\
\hline PE & 0.792 & 0.000 & 4.500 & 4.543 & 0.673 & 0.001 & 4.596 & 4.426 & 0.273 & 0.015 & 4.211 & 4.818 \\
\hline & & & 76 & 221 & & & 57 & 155 & & & 19 & 66 \\
\hline
\end{tabular}




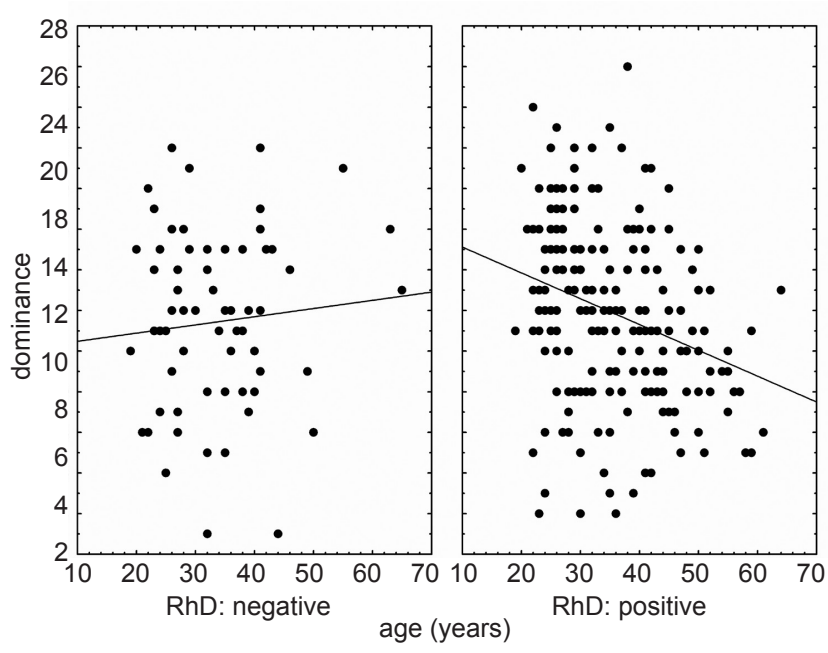

Fig. 1. Correlation between age and Cattell's dominance (E) in $\mathrm{Rh}$-negative and Rh-positive subjects. The statistical significance of the $\mathrm{RhD}$-age interaction estimated by the univariate GLM was 0.006 .

toxoplasmosis $(p=0.137), \mathrm{RhD}$ phenotype $(\mathrm{p}=0.075)$ and toxoplasmosis-RhD interaction $(\mathrm{p}=0.851)$. The univariate GLM analyses performed separately for men and women showed that except for novelty seeking (NS) and self transcendence (ST), the particular effects of toxoplasmosis and RhD phenotype were stronger in men than women (Table 1).

The multivariate GLM with independent factors toxoplasmosis and age showed the effect of age $(p<0.0001)$ and toxoplasmosis $(p=0.025)$ for the whole data set, the effect of age $(p<0.0001)$ and a non-significant effect of toxoplasmosis $(p=0.347)$ for the subset of men, and the effect of age $(p=0.011)$ and toxoplasmosis $(p=0.007)$ for the subset of women. The multivariate GLM analyses with independent factors RhD phenotype and age showed the effect of age (all: $p<0.0001$, men: $p<0.0001$, women: $\mathrm{p}<0.0001)$ and the effect of $\mathrm{RhD}$ phenotype in women (all: $p=0.079$, men: $p<0.149$, women: $p<0.003$ ). Results of particular univariate GLM analyses are shown in Tables 2 and 3 .

\section{Cattell's 16PF}

The multivariate GLM with toxoplasmosis, RhD phenotype and age as three independent factors and sixteen Cattells's factors as dependent variables showed the effects of age $(p<0.0001), R h D$ phenotype $(p=0.020)$, toxoplasmosis $(\mathrm{p}=0.002)$ and toxoplasmosis-RhD interaction $(p<0.001)$. The univariate GLM analyses with independent factors toxoplasmosis, $\mathrm{RhD}$ phenotype and age showed the effect of toxoplasmosis on superego strength $(\mathrm{G})(\mathrm{p}=0.001)$, protension $(\mathrm{L})(\mathrm{p}=0.011)$ and shrewdness (N) $(\mathrm{p}=0.026)$, the effect of $\mathrm{RhD}$ phenotype on ego weakness $(C)(p=0.005)$, protension $(L)(p=0.017)$ and praxernia $(M)(p=0.033)$ and the effect of toxoplasmosis-RhD interaction on ego weakness $(C)(p=0.003)$, praxernia

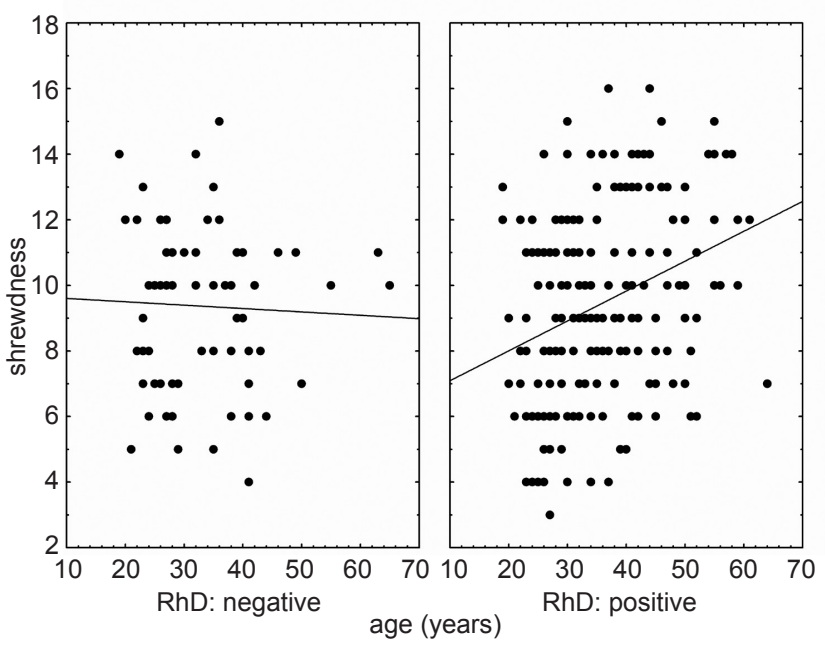

Fig. 2. Correlation between age and Cattell's shrewdness (N) in Rh-negative and Rh-positive subjects. The statistical significance of the RhD-age interaction estimated by the univariate GLM was 0.014 .

(M) $(\mathrm{p}=0.005)$ and ergic tension $\left(\mathrm{Q}_{4}\right)(\mathrm{p}=0.011)$. For the strength and direction of the effects, see Table 4. Latent toxoplasmosis is known to have opposite effects on many Cattell's factors in man and women and therefore we repeated the same analysis separately for men and women. The multivariate GLM analysis performed on the subset of men showed the effects of age $(p<0.0001)$, toxoplasmosis $(p=0.002)$, RhD phenotype $(p=0.002)$ and toxoplasmosis-RhD interaction $(\mathrm{p}<0.001)$. The multivariate GLM analysis performed on the subset of women did not find significant effects of age $(p=0.054)$, toxoplasmosis $(p=0.326)$, RhD phenotype $(p=0.618)$ and toxoplasmosis-RhD interaction $(p=0.851)$. For particular results of the univariate GLM analyses, see Table 4.

The multivariate GLM analyses with independent factors toxoplasmosis and age showed the effect of age (all: $\mathrm{p}<0.0001$, men: $\mathrm{p}<0.0001$, women: $\mathrm{p}<0.0001)$ and no effect of toxoplasmosis (all: $p=0.118$, men: $p=0.088$, women: $p=0.504$ ). The multivariate GLM analyses with independent factors $\mathrm{RhD}$ phenotype and age showed the effect of age (all: $\mathrm{p}<0.0001$, men: $\mathrm{p}<0.0001$, women: $\mathrm{p}<0.0001$ ) and the effect of $\mathrm{RhD}$ phenotype in men (all: $p=0.082$, men: $p=0.036$, women: $p=0.262)$. For particular results of the univariate GLM analyses, see Tables 5 and 6.

To reveal possible protective effects of RhD phenotype against biological factors other than toxoplasmosis, we included the RhD-age interaction into our models. The multivariate GLM analyses showed no significant effect of RhD-age interaction on Cattell's factors (all: $p=0.189$, men: $p=0.326$, women: $p=0.657$ ) or Cloninger's factors (all: $p=0.710$, men: $p=0.434$, women: $p=0.752$ ). However, the univariate GLM analyses showed the effect of $\mathrm{RhD}$ phenotype-age interaction on dominance (E) (all: $p=0.006$, men: $p=0.060$, women: $p=0.024$ ), see Fig. 1, 
Flegr et al.: RhD phenotype and toxoplasmosis-associated changes in personality

Table 4. Effects of latent toxoplasmosis and RhD phenotype on Cattell's personality factors. The table shows the significance (twosided $p)$, effect size $\left(\eta^{2}\right)$ estimated by the univariate GLM and arithmetic means and number of cases in particular subsets. The results significant in two-tailed tests are printed in bold. A - afectothimia/schizothimia, B - low intelligence/high intelligence, C - ego weakness/high ego strength, E - submissiveness/dominance, F - desurgency/surgency, G - low superego strength/high superego strength, $\mathrm{H}$ - threctia/parmia, I - harria/premsia, L - alaxia/protension, $\mathrm{M}$ - praxernia/autia, $\mathrm{N}$ - naivete/shrewdness, $\mathrm{O}$ - untroubled adequacy/guilt proneness, $\mathrm{Q}_{1}$ - conservatism/radicalism, $\mathrm{Q}_{2}$ - group dependency/self sufficiency, $\mathrm{Q}_{3}$ - low self-sentiment integration/high strength of self-sentiment, $\mathrm{Q}_{4}-$ low ergic tension/high ergic tension.

\begin{tabular}{|c|c|c|c|c|c|c|c|c|c|c|c|c|c|c|c|c|c|c|}
\hline & \multicolumn{6}{|c|}{ All } & \multicolumn{6}{|c|}{ Men } & \multicolumn{6}{|c|}{ Women } \\
\hline & $\mathrm{p}$ & $\eta^{2}$ & $\begin{array}{l}\text { toxo- } \\
\mathrm{RhD}^{-}\end{array}$ & $\begin{array}{l}\text { toxo- } \\
\text { RhD }^{+}\end{array}$ & $\begin{array}{l}\text { toxo } \\
\text { RhD }^{-} \\
\end{array}$ & $\begin{array}{l}\text { toxo } \\
\mathrm{RhD}^{+}\end{array}$ & $\mathrm{p}$ & $\eta^{2}$ & $\begin{array}{l}\text { toxo- } \\
\text { RhD- }^{-}\end{array}$ & $\begin{array}{l}\text { toxo- } \\
\mathrm{RhD}^{+}\end{array}$ & $\begin{array}{l}\text { toxo } \\
\mathrm{RhD}^{-} \\
\end{array}$ & $\begin{array}{l}\text { toxo }^{+} \\
\mathrm{RhD}^{+}\end{array}$ & $\mathrm{p}$ & $\eta^{2}$ & $\begin{array}{l}\text { toxo- } \\
\mathrm{RhD}^{-}\end{array}$ & $\begin{array}{l}\text { toxo- } \\
\mathrm{RhD}^{+}\end{array}$ & $\begin{array}{l}\text { toxo } \\
\mathrm{RhD}^{-}\end{array}$ & $\begin{array}{l}\text { toxo }^{+} \\
\mathrm{RhD}^{+}\end{array}$ \\
\hline & 0.618 & 0.00 & 10.707 & 11.060 & 11.857 & 10.403 & 0.245 & 0.01 & 10.000 & 10.217 & 11.556 & 9.958 & 0.927 & 0.00 & 12.071 & 12.951 & 13.667 & 11.526 \\
\hline & 0.359 & 0.00 & & & & & 0.274 & 0.01 & & & & & 0.595 & 0.00 & & & & \\
\hline & 0.101 & 0.01 & & & & & 0.128 & 0.01 & & & & & 0.197 & 0.02 & & & & \\
\hline \multirow[t]{3}{*}{ B } & 0.173 & 0.01 & 8.390 & 8.068 & 7.524 & 7.836 & 0.248 & 0.01 & 8.407 & 8.163 & 7.833 & 7.667 & 0.203 & 0.02 & 8.357 & 7.854 & 5.667 & 8.263 \\
\hline & 0.747 & 0.00 & & & & & 0.856 & 0.00 & & & & & 0.154 & 0.03 & 7.854 & & & \\
\hline & 0.291 & 0.00 & & & & & 0.783 & 0.00 & & & & & 0.045 & 0.05 & & & & \\
\hline & 0.145 & 0.01 & 14.439 & 14.323 & 11.810 & 15.194 & 0.100 & 0.01 & 15.074 & 14.337 & 11.667 & 15.500 & 0.890 & 0.00 & 13.214 & 14.293 & 12.667 & 14.421 \\
\hline & 0.005 & 0.03 & & & & & 0.017 & 0.03 & & & & & 0.298 & 0.02 & & & & \\
\hline & 0.003 & 0.03 & & & & & 0.001 & 0.06 & & & & & 0.800 & 0.00 & & & & \\
\hline \multirow[t]{3}{*}{ E } & 0.976 & 0.00 & 13.171 & 14.053 & 13.762 & 13.358 & 0.848 & 0.00 & 12.444 & 14.370 & 13.444 & 13.708 & 0.916 & 0.00 & 14.571 & 13.341 & 15.667 & 12.474 \\
\hline & 0.449 & 0.00 & & & & & 0.048 & 0.02 & & & & & 0.135 & 0.03 & & & & \\
\hline & 0.501 & 0.00 & & & & & 0.506 & 0.00 & & & & & 0.506 & 0.01 & & & & \\
\hline \multirow[t]{3}{*}{$\mathrm{F}$} & 0.274 & 0.00 & 11.951 & 12.617 & 11.095 & 11.836 & 0.795 & 0.00 & 10.407 & 12.283 & 10.333 & 12.000 & 0.877 & 0.00 & 14.929 & 13.366 & 15.667 & 11.421 \\
\hline & 0.080 & 0.01 & & & & & 0.005 & 0.04 & & & & & 0.062 & 0.05 & & & & \\
\hline & 0.543 & 0.00 & & & & & 0.699 & 0.00 & & & & & 0.400 & 0.01 & & & & \\
\hline & 0.001 & 0.04 & 11.171 & 11.331 & 13.619 & 12.448 & 0.008 & 0.04 & 11.148 & 11.152 & 13.500 & 12.250 & 0.064 & 0.05 & 11.214 & 11.732 & 14.333 & 12.947 \\
\hline & 0.157 & 0.01 & & & & & 0.138 & 0.01 & & & & & 0.630 & 0.00 & & & & \\
\hline & 0.113 & 0.01 & & & & & 0.172 & 0.01 & & & & & 0.356 & 0.01 & & & & \\
\hline & 0.310 & 0.00 & 11.829 & 12.586 & 10.857 & 11.582 & 0.798 & 0.00 & 10.741 & 12.174 & 10.667 & 11.750 & 0.337 & 0.01 & 13.929 & 13.512 & 12.000 & 11.158 \\
\hline & 0.231 & 0.01 & & & & & 0.113 & 0.01 & & & & & 0.804 & 0.00 & & & & \\
\hline & 0.774 & 0.00 & & & & & 0.860 & 0.00 & & & & & 0.946 & 0.00 & & & & \\
\hline \multirow[t]{3}{*}{ I } & 0.708 & 0.00 & 9.439 & 8.977 & 8.143 & 9.806 & 0.792 & 0.00 & 8.037 & 7.848 & 7.333 & 8.917 & 0.533 & 0.01 & 12.143 & 11.512 & 13.000 & 12.053 \\
\hline & 0.406 & 0.00 & & & & & 0.449 & 0.00 & & & & & 0.470 & 0.01 & & & & \\
\hline & 0.117 & 0.01 & & & & & 0.286 & 0.01 & & & & & 0.882 & 0.00 & & & & \\
\hline & 0.011 & 0.03 & 12.098 & 10.511 & 10.524 & 9.597 & 0.135 & 0.01 & 11.222 & 10.565 & 10.611 & 9.521 & 0.052 & 0.05 & 13.786 & 10.390 & 10.000 & 9.789 \\
\hline & 0.017 & 0.02 & & & & & 0.148 & 0.01 & & & & & 0.105 & 0.04 & & & & \\
\hline & 0.406 & 0.00 & & & & & 0.760 & 0.00 & & & & & 0.121 & 0.03 & & & & \\
\hline $\mathrm{M}$ toxo & 0.580 & 0.00 & 11.854 & 11.511 & 10.143 & 12.687 & 0.976 & 0.00 & 11.704 & 11.772 & 10.167 & 13.333 & 0.384 & 0.01 & 12.143 & 10.927 & 10.000 & 11.053 \\
\hline \multirow{2}{*}{$\begin{array}{l}\mathrm{RhD} \\
\text { toxo } \times \mathrm{RhD}\end{array}$} & 0.033 & 0.02 & & & & & 0.007 & 0.04 & & & & & 0.957 & 0.00 & & & & \\
\hline & 0.005 & 0.03 & & & & & 0.008 & 0.04 & & & & & 0.307 & 0.01 & & & & \\
\hline $\mathrm{N}$ toxo & 0.026 & 0.02 & 8.902 & 9.286 & 10.286 & 9.821 & 0.083 & 0.02 & 9.111 & 9.196 & 10.333 & 9.604 & 0.287 & 0.02 & 8.500 & 9.488 & 10.000 & 10.368 \\
\hline RhD & 0.553 & 0.00 & & & & & 0.241 & 0.01 & & & & & 0.554 & 0.00 & & & & \\
\hline toxo $\times \mathrm{RhD}$ & 0.169 & 0.01 & & & & & 0.218 & 0.01 & & & & & 0.706 & 0.00 & & & & \\
\hline O toxo & 0.579 & 0.00 & 10.195 & 10.323 & 11.333 & 9.896 & 0.459 & 0.00 & 9.593 & 9.913 & 11.222 & 9.313 & 0.854 & 0.00 & 11.357 & 11.244 & 12.000 & 11.368 \\
\hline $\mathrm{RhD}$ & 0.297 & 0.00 & & & & & 0.272 & 0.01 & & & & & 0.783 & 0.00 & & & & \\
\hline toxo $\times \mathrm{RhD}$ & 0.215 & 0.01 & & & & & 0.117 & 0.01 & & & & & 0.850 & 0.00 & & & & \\
\hline $\mathrm{Q}_{1}$ toxo & 0.775 & 0.00 & 7.073 & 7.090 & 7.714 & 6.657 & 0.330 & 0.01 & 6.778 & 7.163 & 7.944 & 7.042 & 0.192 & 0.02 & 7.643 & 6.927 & 6.333 & 5.684 \\
\hline RhD & 0.441 & 0.00 & & & & & 0.925 & 0.00 & & & & & 0.497 & 0.01 & & & & \\
\hline toxo $\times \mathrm{RhD}$ & 0.359 & 0.00 & & & & & 0.358 & 0.00 & & & & & 0.924 & 0.00 & & & & \\
\hline $\mathrm{Q}_{2}$ toxo & 0.341 & 0.00 & 12.146 & 11.436 & 10.810 & 11.731 & 0.071 & 0.02 & 12.630 & 12.065 & 11.000 & 11.458 & 0.749 & 0.00 & 11.214 & 10.024 & 9.667 & 12.421 \\
\hline $\mathrm{RhD}$ & 0.932 & 0.00 & & & & & 0.840 & 0.00 & & & & & 0.551 & 0.00 & & & & \\
\hline toxo $\times \mathrm{RhD}$ & 0.166 & 0.01 & & & & & 0.460 & 0.00 & & & & & 0.134 & 0.03 & & & & \\
\hline $\mathrm{Q}_{3}$ toxo & 0.652 & 0.00 & 11.707 & 11.977 & 11.381 & 12.761 & 0.796 & 0.00 & 12.222 & 12.152 & 11.222 & 12.854 & 0.274 & 0.02 & 10.714 & 11.585 & 12.333 & 12.526 \\
\hline RhD & 0.136 & 0.01 & & & & & 0.211 & 0.01 & & & & & 0.668 & 0.00 & & & & \\
\hline toxo $\times \mathrm{RhD}$ & 0.320 & 0.00 & & & & & 0.158 & 0.01 & & & & & 0.720 & 0.00 & & & & \\
\hline $\mathrm{Q}_{4}$ toxo & 0.145 & 0.01 & 12.024 & 12.910 & 14.810 & 12.104 & 0.013 & 0.03 & 10.815 & 12.543 & 15.444 & 11.729 & 0.208 & 0.02 & 14.357 & 13.732 & 11.000 & 13.053 \\
\hline $\mathrm{RhD}$ & 0.209 & 0.01 & & & & & 0.217 & 0.01 & & & & & 0.655 & 0.00 & & & & \\
\hline toxo $\times \mathrm{RhD}$ & 0.011 & 0.03 & & & & & 0.001 & 0.06 & & & & & 0.402 & 0.01 & & & & \\
\hline $\mathrm{n}$ & & & 41 & 133 & 21 & 67 & & & 27 & 92 & 18 & 48 & & & 14 & 41 & 3 & 19 \\
\hline
\end{tabular}


Table 5. Effects of latent toxoplasmosis on Cattell's personality factors. The table shows the significance (two-sided p), effect size $\left(\eta^{2}\right)$ estimated by the univariate GLM and arithmetic means in particular subsets. The results significant in two-tailed tests are printed in bold. For abbreviations see Table 4.

\begin{tabular}{|c|c|c|c|c|c|c|c|c|c|c|c|c|}
\hline & \multicolumn{4}{|c|}{ All } & \multicolumn{4}{|c|}{ Men } & \multicolumn{4}{|c|}{ Women } \\
\hline & $\mathrm{p}$ & $\eta^{2}$ & toxo- & toxo ${ }^{+}$ & $\mathrm{p}$ & $\eta^{2}$ & toxo- & toxo $^{+}$ & $\mathrm{p}$ & $\eta^{2}$ & toxo- & toxo $0^{+}$ \\
\hline A & 0.707 & 0.001 & 10.942 & 10.778 & 0.577 & 0.002 & 10.139 & 10.406 & 0.287 & 0.015 & 12.740 & 11.843 \\
\hline B & 0.304 & 0.004 & 8.141 & 7.802 & 238 & 0.007 & 8.201 & 7.728 & 948 & 0.000 & 8.002 & 8.041 \\
\hline C & 0.930 & 0.000 & 14.351 & 14.396 & 922 & 0.000 & 14.521 & 14.461 & 0.828 & 0.001 & 13.978 & 14.193 \\
\hline E & 679 & 0.001 & 13.771 & 13.537 & 833 & 0.000 & 13.828 & 13.687 & 0.490 & 0.006 & 13.694 & 12.961 \\
\hline $\mathrm{F}$ & 261 & 0.005 & 12.468 & 11.805 & 0.662 & 0.001 & 11.913 & 11.603 & 0.212 & 0.021 & 13.698 & 12.405 \\
\hline G & 0.005 & 0.030 & 11.335 & 12.655 & 0.018 & 0.029 & 11.189 & 12.554 & 0.084 & 0.039 & 11.640 & 13.008 \\
\hline $\mathrm{H}$ & 0.279 & 0.004 & 12.372 & 11.511 & 0.719 & 0.001 & 11.845 & 11.503 & 0.149 & 0.028 & 13.571 & 11.456 \\
\hline I & 0.581 & 0.001 & 9.080 & 9.382 & 0.397 & 0.004 & 7.932 & 8.461 & 0.489 & 0.006 & 11.613 & 12.167 \\
\hline $\mathrm{L}$ & 0.014 & 0.023 & 10.863 & 9.847 & 0.061 & 0.019 & 10.722 & 9.826 & 0.137 & 0.029 & 11.168 & 9.937 \\
\hline M & 0.250 & 0.005 & 11.579 & 12.066 & 0.129 & 0.012 & 11.708 & 12.458 & 0.688 & 0.002 & 11.272 & 10.944 \\
\hline $\mathrm{N}$ & 0.058 & 0.013 & 9.200 & 9.876 & 0.151 & 0.011 & 9.182 & 9.779 & 0.192 & 0.023 & 9.237 & 10.170 \\
\hline $\mathrm{O}$ & 0.987 & 0.000 & 10.249 & 10.240 & 0.889 & 0.000 & 9.755 & 9.839 & 1.000 & 0.000 & 11.372 & 11.370 \\
\hline $\mathrm{Q}_{1}$ & 0.728 & 0.000 & 7.091 & 6.961 & 0.643 & 0.001 & 7.100 & 7.311 & 0.066 & 0.044 & 7.077 & 5.896 \\
\hline $\mathrm{Q}_{2}$ & 0.904 & 0.000 & 11.548 & 11.492 & 0.136 & 0.012 & 12.117 & 11.325 & 0.069 & 0.044 & 10.274 & 12.030 \\
\hline $\mathrm{Q}_{3}$ & 0.242 & 0.005 & 11.916 & 12.398 & 0.657 & 0.001 & 12.183 & 12.399 & 0.178 & 0.024 & 11.331 & 12.385 \\
\hline$V_{2}$ & 0.935 & 0.000 & 12.723 & 12.771 & 0.404 & 0.004 & 12.188 & 12.753 & 0.316 & 0.013 & 13.929 & 12.773 \\
\hline $\mathrm{n}$ & & & 180 & 8 & & & 124 & 66 & & & 56 & 22 \\
\hline
\end{tabular}

and shrewdness $(\mathrm{N})$ (all: $\mathrm{p}=0.014$, men: $\mathrm{p}=0.007$, women: $p=0.558)$, see Fig. 2 .

As a sort of negative control, we repeated all analyses with $\mathrm{ABO}$ phenotype used instead of RhD phenotype. The multivariate GLM analysis revealed no significant effect of $\mathrm{ABO}$ phenotype or $\mathrm{ABO}$ phenotype-toxoplasmosis interaction (results not shown). Of 69 univariate analyses (7 Cloninger's and 16 Cattell's factors, all subjects, men, women), only two, the tests of reward dependence (RD) in all subjects $(\mathrm{p}=0.048)$ and premsia (I) in all subjects $(p=0.029)$, revealed significant effects of the studied factors (ABO phenotype-toxoplasmosis interaction). These effects were not significant after the Bonferroni correction for multiple tests.

\section{DISCUSSION}

"Asymptomatic" latent toxoplasmosis and $\mathrm{RhD}$ phenotype, i.e. the presence or absence of the $\mathrm{D}$ antigen on the membrane of erythrocytes, had a specific effect on the personality profile of blood donors estimated by Cattell's 16PF questionnaire and Cloninger's TCI questionnaire. Moreover, toxoplasmosis had both quantitatively and qualitatively different effects on the personality profile of Rh-positive and Rh-negative subjects.

The specific influence of latent toxoplasmosis on Cloninger's factors has already been described in a nearly identical set of blood donors for novelty seeking (NS) and self transcendence (ST) (Skallová et al. 2005) and in an independent set of military conscripts for novelty seeking (Novotná et al. 2005). The analysis of the statistical models containing both toxoplasmosis and $\mathrm{Rh}$ phenotype as independent factors identified not only possible effects of RhD phenotype on harm avoidance (HA), cooperativeness (CO) and self transcendence (ST) (in women) but also opposite effects of toxoplasmosis on reward dependence (RD) and cooperativeness (CO) in Rh-positive and Rh-negative subjects. Similarly, specific effects of latent toxoplasmosis on Cattell's personality factors strength of superego $(\mathrm{G})$ and protension (L) have already been reported in university students (Flegr and Hrdý 1994), military conscripts (Novotná et al. 2005), blood donors (Skallová et al. 2005), childbearing age women (Flegr and Havlíček 1999) and males (Flegr et al. 1996) and females (Flegr et al. 2000) diagnosed with acute toxoplasmosis 2-12 years before personality testing, (see Flegr 2007 for review). Again, the current analysis of the statistical models containing both toxoplasmosis and $\mathrm{Rh}$ phenotype as independent factors identified possible effects of RhD phenotype on ego strength (C), protension (L), and praxernia (M) and opposite effects of toxoplasmosis on ego strength $(C)$, praxernia $(M)$, ergic tension $\left(Q_{4}\right)$. and cooperativeness $(\mathrm{CO})$ in $\mathrm{Rh}$-positive and $\mathrm{Rh}$-negative subjects. It should be stressed that not only toxoplasmosis-RhD interaction but also some of the main effects of toxoplasmosis and nearly all of the main effects of RhD phenotype can only be proved to exist in the models containing both analyzed factors, i.e. toxoplasmosis and $\mathrm{RhD}$ phenotype. Therefore, the previous unsuccessful attempts to prove the correlation between various personality traits and blood groups (Irvine and Miyashit 1965, Wiener 1965, Cattell 1972, Rogers and Glendon 2003, Wu et al. 2005) can be explained not only by the fact that the $\mathrm{ABO}$ rather than the $\mathrm{Rh}$ factor blood group system was usually studied, but also by the fact that the important confounding factor, toxoplasmosis, was not included in the analyzed models.

Four studies have already shown that the response of the human body to latent toxoplasmosis depends on RhD phenotype (Flegr et al. 2008, 2009, Novotná et al. 
Table 6. Effects of RhD phenotype on Cattell's personality factors. The table shows the significance (two-sided p), effect size ( $\eta^{2}$ ) estimated by the univariate GLM and arithmetic means in particular subsets. The results significant in two-tailed tests are printed in bold. For abbreviations see Table 4.

\begin{tabular}{|c|c|c|c|c|c|c|c|c|c|c|c|c|}
\hline & \multicolumn{4}{|c|}{ All } & \multicolumn{4}{|c|}{ Men } & \multicolumn{4}{|c|}{ Women } \\
\hline & $p$ & $\eta^{2}$ & $\mathrm{RhD}^{-}$ & $\mathrm{RhD}^{+}$ & $p$ & $\eta^{2}$ & $\mathrm{RhD}^{-}$ & $\mathrm{RhD}^{+}$ & $p$ & $\eta^{2}$ & $\mathrm{RhD}^{-}$ & $\mathrm{RhD}^{+}$ \\
\hline A & 0.727 & 0.000 & 11.005 & 10.839 & 0.420 & 0.003 & 10.552 & 10.127 & 0.856 & 0.000 & 12.337 & 12.505 \\
\hline B & 0.896 & 0.000 & 7.983 & 8.030 & 0.989 & 0.000 & 8.047 & 8.040 & 0.780 & 0.001 & 7.823 & 8.000 \\
\hline $\mathrm{C}$ & 0.081 & 0.011 & 13.637 & 14.612 & 0.163 & 0.010 & 13.809 & 14.733 & 0.253 & 0.018 & 13.109 & 14.336 \\
\hline E & 0.298 & 0.004 & 13.261 & 13.899 & 0.028 & 0.026 & 12.682 & 14.274 & 0.145 & 0.028 & 14.742 & 13.073 \\
\hline F & 0.101 & 0.010 & 11.415 & 12.471 & 0.006 & 0.039 & 10.222 & 12.327 & 0.064 & 0.045 & 14.866 & 12.805 \\
\hline G & 0.413 & 0.003 & 12.061 & 11.642 & 0.244 & 0.007 & 12.169 & 11.438 & 0.762 & 0.001 & 11.833 & 12.097 \\
\hline $\mathrm{H}$ & 0.275 & 0.005 & 11.353 & 12.297 & 0.143 & 0.011 & 10.583 & 12.095 & 0.665 & 0.003 & 13.491 & 12.794 \\
\hline I & 0.742 & 0.000 & 9.027 & 9.225 & 0.737 & 0.001 & 7.934 & 8.165 & 0.473 & 0.007 & 12.302 & 11.681 \\
\hline $\mathrm{L}$ & 0.006 & 0.028 & 11.470 & 10.234 & 0.186 & 0.009 & 10.935 & 10.235 & 0.001 & 0.134 & 13.058 & 10.217 \\
\hline M & 0.279 & 0.004 & 11.383 & 11.884 & 0.064 & 0.018 & 11.269 & 12.268 & 0.378 & 0.011 & 11.750 & 10.971 \\
\hline $\mathrm{N}$ & 0.823 & 0.000 & 9.489 & 9.402 & 0.301 & 0.006 & 9.724 & 9.252 & 0.241 & 0.019 & 8.836 & 9.746 \\
\hline $\mathrm{O}$ & 0.743 & 0.000 & 10.385 & 10.192 & 0.716 & 0.001 & 9.978 & 9.738 & 0.843 & 0.001 & 11.515 & 11.271 \\
\hline $\mathrm{Q}_{1}$ & 0.606 & 0.001 & 7.191 & 6.982 & 0.933 & 0.000 & 7.127 & 7.169 & 0.258 & 0.017 & 7.348 & 6.551 \\
\hline $\mathrm{Q}_{2}$ & 0.691 & 0.001 & 11.738 & 11.534 & 0.795 & 0.000 & 12.007 & 11.856 & 0.868 & 0.000 & 10.956 & 10.779 \\
\hline $\mathrm{Q}_{3}$ & 0.203 & 0.006 & 11.657 & 12.222 & 0.316 & 0.005 & 11.852 & 12.378 & 0.337 & 0.012 & 11.055 & 11.868 \\
\hline $\mathrm{Q}_{4}$ & 0.659 & 0.001 & 12.931 & 12.649 & 0.627 & 0.001 & 12.640 & 12.281 & 0.847 & 0.001 & 13.761 & 13.518 \\
\hline $\mathrm{n}$ & & & 66 & 201 & & & 49 & 141 & & & 17 & 60 \\
\hline
\end{tabular}

2008, Kaňková et al. 2010). Psychomotor performance (reaction times) of Rh-negative subjects deteriorated after Toxoplasma infection while Rh-positive heterozygotes were permanently and Rh-positive homozygotes were temporarily protected against such decrease in psychomotor performance. Also, RhD-negative women with latent toxoplasmosis gained more weight during pregnancy than RhD-negative, Toxoplasma-free women or RhDpositive women. Results of the present study suggest that RhD phenotype also modulates toxoplasmosis-associated changes in the personality profile. In contrast to the primarily quantitative modulation of changes in reaction times, the modulation of some personality profile changes was also qualitative. For example, latent toxoplasmosis was associated with lower ego strength and lower cooperativeness in Rh-negative subjects, but with higher ego strength and higher cooperativeness in Rh-positive subjects. The toxoplasmosis-associated changes were always stronger in Rh-negative subjects, as was reported previously for psychomotor performance.

Our present results indicate that $\mathrm{RhD}$ phenotype modulates the responses of the body not only to Toxoplasma infection, but also to other factors. Here we observed different correlations between two Cattell's factors, namely dominance and shrewdness, and age in Rh-positive and Rh-negative subjects. In contrast to the response of the body to Toxoplasma infection, the correlation with the personality factors was stronger in Rh-positive subjects. It must be pointed out, however, that unlike the effect of RhD-toxoplasmosis interaction, the effect of RhD-age interaction on Cattell's personality profile estimated by the multivariate GLM was not significant and the significances in the univariate tests did not survive the Bonferroni correction. Therefore, the significant effects of $\mathrm{RhD}$-age interaction observed in two of sixteen univariate GLM tests might be just artifacts of the multiple statistical tests and should be confirmed in other independent studies.

The function of the $\mathrm{RhD}$ molecule, the membrane pump that transports either ammonium or $\mathrm{CO}_{2}$, has been revealed only recently, but its physiological role remains unknown (Biver et al. 2006, Kustu and Inwood 2006). Therefore, the speculation about the mechanisms involved in the modulation of toxoplasmosis- (or age-) associated changes in personality traits (or reaction times or weight gain) would be rather premature. Obviously, the difference between Rh-positive homozygotes and heterozygotes, namely permanent protection against the impairment of reaction times in heterozygotes and only temporary protection in homozygytes (Novotná et al. 2008), suggests that not (only) the $\mathrm{RhD}$ molecule but (also) a product of another gene in a strong genetic linkage with the $R H D$ gene might be responsible for the observed biological and psychological effects of RhD phenotype (Flegel 2006). To test such hypotheses, genetic instead of common serological data needs to be collected from larger populations.

The origin and maintenance of the Rh polymorphism in the human population is an old evolutionary enigma, as the fitness of carriers of the rarer variant of the RHD gene (an Rh-negative woman in a predominantly Rh-positive population or an Rh-positive man in a predominantly Rh-negative population) is compromised by the risk of haemolytic disease of newborns (Fisher et al. 1944, Haldane 1944). The broad spectrum of behavioural effects of $\mathrm{RhD}$ phenotype described in this and previous studies, however, suggests that the Rh polymorphism can be stabilized by some form of frequency dependent selection.

Acknowledgements. This work was supported by grant No. 0021 620828 from the Czech Ministry of Education, Youth and Sports. 


\section{REFERENCES}

Biver S., Scohy S., Szpirer J., Szpirer C., Andre B., Marini A.M. 2006: Physiological role of the putative ammonium transporter RhCG in the mouse. Transfus. Clin. Biol. 13: 167-168.

Carritt B., Kemp T.J., Poulter M. 1997: Evolution of the human RH (rhesus) blood group genes: a 50 year old prediction (partially) fulfilled. Hum. Mol. Gen. 6: 843-850.

Cattell R.B. 1972: Blood-groups and personality traits. Am. J. Hum. Gen. 24: 485-486.

Cloninger C.R., Przybeck T.R., Svrakic D.M., Wetzel R.D. 1994: The temperament and character inventory (TCI): a guide to its development and use. Washington University Press, Center for Psychobiology of Personality, St. Louis, Missouri, 184 pp.

Fisher R.A., Race R.R., TAYlor G.L. 1944: Mutation and the Rhesus reaction. Nature 153: 106.

Flegel W.A. 2006: Molecular genetics of RH and its clinical application. Transfus. Clin. Biol. 13: 4-12.

Flegr J. 2007: Effects of Toxoplasma on human behavior. Schizophr. Bull. 33: 757-760.

Flegr J., Havlíčé J. 1999: Changes in the personality profile of young women with latent toxoplasmosis. Folia Parasitol. 46: 22-28.

Flegr J., HavlíčeK J., Kodym P., Malý M., ŠMahel Z. 2002: Increased risk of traffic accidents in subjects with latent toxoplasmosis: a retrospective case-control study. BMC Infect. Dis. 2: e11.

FLEGR J., HRdÝ I. 1994: Influence of chronic toxoplasmosis on some human personality factors. Folia Parasitol. 41: 122-126.

Flegr J., Klose J., Novotná M., Berenreitterová M., Havlíček J. 2009: Increased incidence of traffic accidents in Toxoplasmainfected military drivers and protective effect $\mathrm{RhD}$ molecule revealed by a large-scale prospective cohort study. BMC Infect. Dis. 9: e72.

Flegr J., Kodym P., Tolarová V. 2000: Correlation of duration of latent Toxoplasma gondii infection with personality changes in women. Biol. Psychol. 53: 57-68.

Flegr J., Novotná M., Lindová J., Havlíček J. 2008: Neurophysiological effect of the $\mathrm{Rh}$ factor. Protective role of the RhD molecule against Toxoplasma-induced impairment of reaction times in women. Neuroendocrinol. Lett. 29: 475-481.

Flegr J., Zitková S., Kodym P., Frynta D. 1996: Induction of changes in human behaviour by the parasitic protozoan Toxoplasma gondii. Parasitology 113: 49-54.

Gaskell E.A., Smith J.E., Pinney J.W., Westhead D.R., McCoNKEY G.A. 2009: A unique dual activity amino acid hydroxylase in Toxoplasma gondii. PLoS ONE 4: e4801.

Haldane J.B.S. 1944: Mutation and the Rhesus reaction. Nature 153: 106

Havlíček J., Gašová Z., Smith A.P., Zvára K., Flegr J. 2001: Decrease of psychomotor performance in subjects with latent 'asymptomatic' toxoplasmosis. Parasitology 122: 515-520.

Hodková H., Kodym P., Flegr J. 2007: Poorer results of mice with latent toxoplasmosis in learning tests: impaired learning processes or the novelty discrimination mechanism? Parasitology 134: 1329-1337.

Irvine D.G., Miyashit H. 1965: Blood types in relation to depressions and schizophrenia - a preliminary report. Can. Med. Assoc. J. 92: 551.

Jones J.L., Kruszon-Moran D., Wilson M., McQuillan G., Navin T., Mcauley J.B. 2001: Toxoplasma gondii infection in the United States: seroprevalence and risk factors. Am. J. Epidemiol. 154: 357-365.

KAŇKovÁ Š., ŠulC J., Flegr J. 2010: Increased pregnancy weight gain in women with latent toxoplasmosis and $\mathrm{RhD}$-positivity protection against this effect. Parasitology (in press).

Kocazeybek B., Oner Y.A., Turksoy R., Babur C., Cakan H., Sahip N., Unal A., Ozaslan A., Kilic S., Saribas S., Asian M., Taylan A., Koc S., Dirican A., Uner H.B., Oz V., Ertekin C., Kucukbasmaci O., Torun M.M. 2009: Higher prevalence of toxoplasmosis in victims of traffic accidents suggest increased risk of traffic accident in Toxoplasma-infected inhabitants of Istanbul and its suburbs. Forensic Sci. Int. 187: 103-108.

KoŽEnÝ J., TisAnSKÁ L. 1998: [Attributes of temperament and character inventory TCI in a sample of adolescents.] Českosl. Psychol. 42: 75-83. (In Czech.)

Kustu S., Inwood W. 2006: Biological gas channels for $\mathrm{NH}_{3}$ and $\mathrm{CO}_{2}$ : evidence that $\mathrm{Rh}$ (Rhesus) proteins are $\mathrm{CO}_{2}$ channels. Transfus. Clin. Biol. 13: 103-110.

Markell E.K., John D.T., Krotoski W.A. 1999: Markell and Voge's Medical parasitology. W.B. Saunders Company, Philadelphia.

Novotná M., Hanusova J., Klose J., Preiss M., Havlicek J., RouBAlová K., Flegr J. 2005: Probable neuroimmunological link between Toxoplasma and cytomegalovirus infections and personality changes in the human host. BMC Infect. Dis. 5: e54.

Novotná M., Havlíček J., Smith A.P., Kolbeková P., Skallová A., Klose J., Gašová Z., PísačKa M., Sechovská M., Flegr J. 2008: Toxoplasma and reaction time: role of toxoplasmosis in the origin, preservation and geographical distribution of $\mathrm{Rh}$ blood group polymorphism. Parasitology 135: 1253-1261.

Pokorný J., Fruhbauer Z., Poledñáková S., Sýkora J., ZÁstěra M., Fialová D. 1989: [Assessment of antitoxoplasmatic IgG antibodies with the ELISA method.] Českosl. Epidemiol. Mikrobiol. Imunol. 38: 355-361. (In Czech.)

Preiss M., Klose J. 2003: Diagnostika poruch osobnosti pomocí teorie C R Cloningera. Psychiatrie 4: 226-231.

Remington J.S., Krahenbuhl J.L. 1982: Immunology of Toxoplasma gondii. In: A.J. Nahmias, J. O’Reilly (Eds.), Immunology of Human Infection, Part II. Plenum Publishing Corporation, New York, pp. 327-371.

Roberts L.S., Janovy J. Jr. 2000: Gerald S. Schmidt \& Larry S Roberts' Foundations of Parasitology. 6th Edition. McGraw-Hill Companies, Inc., Boston, 704 pp.

Rogers M., Glendon A.I. 2003: Blood type and personality. Pers. Indiv. Differ. 34: 1099-1112.

Skallová A., Kodym P., Frynta D., Flegr J. 2006: The role of dopamine in Toxoplasma-induced behavioural alterations in mice: an ethological and ethopharmacological study. Parasitology 133: 525-535.

Skallová A., Novotná M., Kolbeková P., Gašová Z., Veselý V., FLEGR J. 2005: Decreased level of novelty seeking in blood donors infected with Toxoplasma. Neuroendocrinol. Lett. 26: 480-486.

Tenter A.M., Heckeroth A.R., Weiss L.M. 2000: Toxoplasma gondii: from animals to humans. Int. J. Parasitol. 30: 1217-1258.

Warren J., Sabin A.B. 1942: The complement fixation reaction in toxoplasmic infection. Proc. Soc. Exp. Biol. Med. 51: 11-16.

Webster J.P. 2001: Rats, cats, people and parasites: the impact of latent toxoplasmosis on behaviour. Microb. Infect. 3: 1037-1045.

Wiener A.S. 1965: Blood groups and personality traits. Am. J. Hum. Gen. 17: 369-370.

Wu K.H., Lindsted K.D., Lee J.W. 2005: Blood type and the five factors of personality in Asia. Pers. Indiv. Differ. 38: 797-808.

Yereli K., Balcioglu I.C., OzBilgin A. 2006: Is Toxoplasma gondii a potential risk for traffic accidents in Turkey? Forensic Sci. Int. 163: 34-37.

Accepted 30 April 2010 